\title{
The Janus-faced chromophore: A donor-acceptor dyad with dual performance in photon up-conversion
}

\author{
Natalia Kiseleva \\ Karlsruhe Institute of Technology \\ Mikhail Filatov \\ Technological University Dublin, mikhail.filatov@tudublin.ie \\ Michael Oldenburg \\ Karlsruhe Institute of Technology
}

See next page for additional authors

Follow this and additional works at: https://arrow.tudublin.ie/scschcpsart

Part of the Chemistry Commons

\section{Recommended Citation}

Kiseleva N , Filatov MA , Oldenburg M , Busko D , Jakoby M , Howard IA, Richards BS , Senge MO , Borisov $S M$, Turshatov A. The Janus-faced chromophore: a donor-acceptor dyad with dual performance in photon up-conversion. Chem Commun (Camb). 2018 Feb 8;54(13):1607-1610. doi: 10.1039/c7cc08930a. PMID: 29369311.

This Article is brought to you for free and open access by the School of Chemical and Pharmaceutical Sciences at ARROW@TU Dublin. It has been accepted for inclusion in Articles by an authorized administrator of ARROW@TU Dublin. For more information, please contact arrow.admin@tudublin.ie, aisling.coyne@tudublin.ie, gerard.connolly@tudublin.ie.

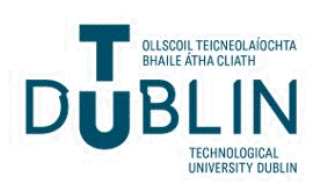




\section{Authors}

Natalia Kiseleva, Mikhail Filatov, Michael Oldenburg, Dimitry Busko, Marius Jakoby, Ian Howard, Bryce Richards, Mathias Senge, Sergey Borisov, and Andrey Turshatov 
Check for updates

Cite this: Chem. Commun., 2018, 54, 1607

Received 20th November 2017,

Accepted 24th December 2017

DOI: $10.1039 / c 7 c c 08930 a$

rsc.li/chemcomm

\section{The Janus-faced chromophore: a donor-acceptor dyad with dual performance in photon up-conversion $\dagger$}

\author{
Natalia Kiseleva, ${ }^{a}$ Mikhail A. Filatov, (D) $\star^{*^{b}}$ Michael Oldenburg, ${ }^{a}$ Dmitry Busko, ${ }^{a}$ \\ Marius Jakoby, ${ }^{a}$ Ian A. Howard, (ID ac Bryce S. Richards, (iD ac Mathias O. Senge, (iD b \\ Sergey M. Borisov (iD d and Andrey Turshatov (D) *a
}

An electron donor-acceptor dyad based on BODIPY (acceptor) and anthracene (donor) plays either the role of sensitizer or emitter in triplet-triplet annihilation photon up-conversion (TTA-UC). This Janus-like behavior was achieved via altering the relative ordering of charge-transfer and local excited state energies in the dyad through the polarity of TTA-UC media.

Triplet-triplet annihilation up-conversion (TTA-UC) ${ }^{1}$ has attracted much attention during the past decade and found applications in material science, ${ }^{2}$ solar energy conversion, ${ }^{3}$ solar fuels, ${ }^{4}$ and bioimaging. ${ }^{5}$ The TTA-UC process takes place in multi-chromophore systems consisting of energetically optimized pairs of sensitizer and emitter molecules. This unique process allows for the emission of high-energy photons from lower-energy excitation photons with high photoluminescence quantum yield (PLQY) at very low intensity and extremely low spectral power density of the optical source used, allowing for use of sunlight. ${ }^{6}$ Generally, a TTA-UC system requires two distinct molecular components: a sensitizer that is excited to form the triplet state and transfer energy to the triplet state of a second component: an emitter. The emitter is capable of forming an emitting singlet state via bimolecular triplet-triplet annihilation with energy higher than the one used to excite the sensitizer.

One of the major challenges in the design of TTA-UC systems is to integrate the sensitizer and emitter functions into a single molecule architecture, which would open up new potential applications.

${ }^{a}$ Institute of Microstructure Technology, Karlsruhe Institute of Technology, Hermann-von-Helmholtz-Platz 1, 76344, Eggenstein-Leopoldshafen, Germany. E-mail: andrey.turshatov@kit.edu

${ }^{b}$ School of Chemistry, SFI Tetrapyrrole Laboratory, Trinity Biomedical Sciences Institute, Trinity College Dublin, The University of Dublin, 152-160 Pearse Street, Dublin 2, Ireland.E-mail: mikhail.filatov@dit.ie

${ }^{c}$ Light Technology Institute, Karlsruhe Institute of Technology, Engesserstrasse 13, 76131 Karlsruhe, Germany

${ }^{d}$ Institute of Analytical Chemistry and Food Chemistry,

Graz University of Technology, Stremayrgasse 9, 8010, Graz, Austria

$\dagger$ Electronic supplementary information (ESI) available: Transient absorption spectra, TTA-UC decays, PdTBTFP phosphorescence decays. See DOI: 10.1039/c7cc08930a \$ New address: School of Chemical and Pharmaceutical Science, Dublin Institute of Technology, Kevin Street, Dublin 8, Ireland.
Attempts to realize such materials based on dimers, ${ }^{7}$ core-shell molecules, ${ }^{8}$ dendrimers and oligomers ${ }^{9}$ have been reported. However, a question of fundamental importance for photonic applications arises from these studies: can a molecule behave both as an efficient emitter fluorophore and as a triplet excited state sensitizer? It is well-known that intersystem crossing (ISC) in a chromophore competes with emission from the singlet excited state. Efficient ISC is a loss channel leading to a dramatic reduction in lifetime and fluorescence quantum yield. ${ }^{10}$ While examples of the chromophores simultaneously exhibiting fluorescence and phosphorescence emission have been reported, the only known general approach to control these functions of the dyes is based on thermally activated delayed fluorescence. ${ }^{11}$

We have recently demonstrated that photo-induced electron transfer (PeT) in BODIPY donor-acceptor dyads allows to switch between strong fluorescence emission and efficient ISC processes, depending on the polarity of the solvent in which they are dissolved. ${ }^{12}$ As shown in Fig. 1 for an exemplary BODIPY-anthracene dyad (BAD), in a non-polar solvent the dyad behaves as a typical fluorophore, possessing high fluorescence quantum yield (up to 0.9) and negligible ISC. This can be explained via the relative ordering of the excited states. In non-polar media a charge-transfer $\left(\mathrm{S}^{\mathrm{CT}}\right)$ state of the dyad is significantly higher in energy than the local excited state of the BODIPY $\left(\mathrm{S}^{\mathrm{BDP}}\right)$; this makes the PeT process inefficient.
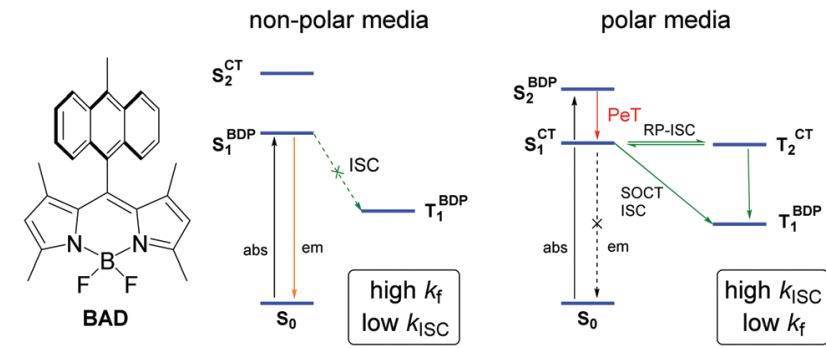

Fig. 1 Photophysics of the BAD molecule in different media. In a nonpolar media the BAD demonstrates high fluorescence rate $\left(k_{\mathrm{f}}\right)$ and low ISC rate $\left(k_{\mathrm{ISC}}\right)$ values. In a polar media - low fluorescence rate $\left(k_{\mathrm{f}}\right)$ and high ISC rate $\left(k_{I S C}\right)$ values. 
Alternatively, in polar solvents, a charge-transfer state of the dyad is stabilized due to electrostatic interactions with the media, resulting in its energy level being below that of $S^{\mathrm{BDP}}$, thus driving the PeT process. The populated charge transfer state can further recombine into a local triplet state of either the donor or acceptor subunits via spin-orbit charge transfer (SOCT) or a radical-pair intersystem crossing (RP-ISC) mechanisms. The high yield of triplet states in these processes and their long lifetimes allow for practical applications involving triplet state formation. For instance, singlet oxygen quantum yield $\left(\Phi_{\Delta}=0.67 \text { in ethanol }\right)^{12}$ of $\mathrm{BAD}$ is comparable or even higher than for conventional triplet sensitizers (e.g. Rose Bengal and methylene blue possess $\Phi_{\Delta}$ of 0.86 and 0.52 , respectively), that allows application of such dyads in photodynamic therapy.

We hypothesize that such a combination of photophysical properties could allow BAD to function either as sensitizer or emitter in TTA-UC. Such functionality could be realized via combining BAD with a second component that possesses appropriate singlet and triplet excited state energies, and an adjustment of the polarity of the media to block/allow the PeT process. With this in mind, we studied the behavior of two systems: (i) BAD combined with perylene in dichloromethane (DCM, $\varepsilon=8.93$ ); and (ii) BAD combined with $\{5,10,15,20$-tetrakis(4-fluorophenyl)tetrabenzoporphyrinato $\}$ palladium(II) (PdTBTFP) in toluene $(\varepsilon=2.38)$. In the first system, BAD should act as the sensitizer, whereas in the second it should act as the emitter. This dual performance of BAD is schematically illustrated in Fig. 2 and has not been demonstrated for any other dye so far.

The precise details explaining the physical basis for UC in the proposed systems are discussed in the following. Fig. 3a shows the absorption spectrum $\left(\lambda_{\max }=505 \mathrm{~nm}\right)$ of BAD dissolved in DCM together with the emission profile of a laser diode used for UC excitation. Fig. 3b displays the UC emission spectra of the $\mathrm{BAD}$-perylene pair dissolved in deoxygenated DCM with different molar ratios of $\mathrm{BAD}$ and perylene. Upon excitation at $525 \mathrm{~nm}$, the solutions exhibit anti-Stokes blue emission of perylene centered at $445 \mathrm{~nm}$. This result confirms that a triplet-triplet energy transfer TTET from the $\mathrm{T}_{1}$ state of BAD to the $\mathrm{T}_{1}$ state of perylene, followed by
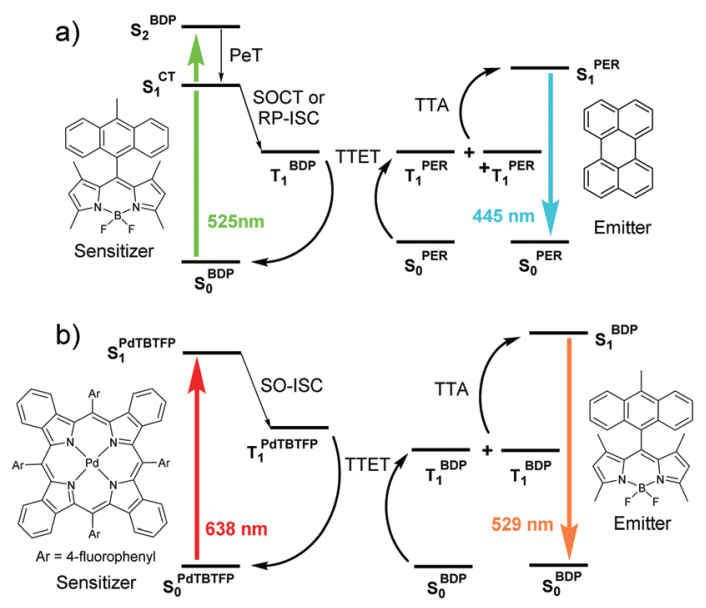

Fig. 2 Photophysical processes taking place in: (a) an UC system employing $B A D$ as a sensitizer and perylene (PER) as an emitter; (b) UC system based on PdTBTFP as a sensitizer and BAD as an emitter.
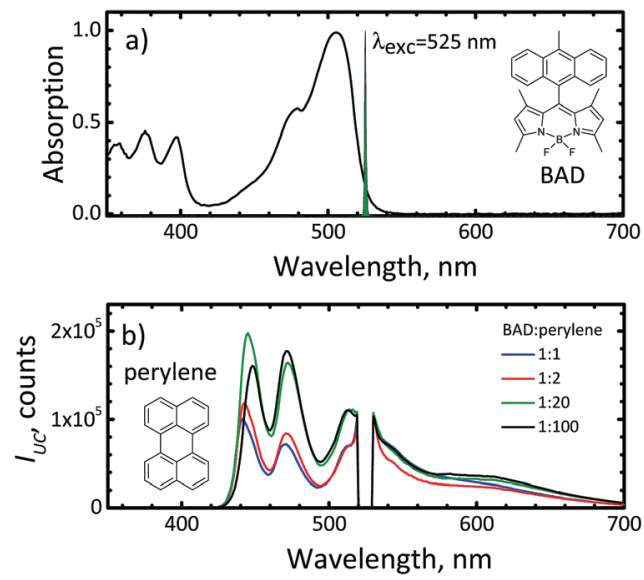

Fig. 3 (a) Normalized absorption spectrum of BAD in DCM and emission profile of a laser diode $(525 \mathrm{~nm})$ used for UC excitation; (b) UC luminescence of perylene under excitation with the $525 \mathrm{~nm}$ laser diode (power density of $5 \mathrm{~W} \mathrm{~cm}^{-2}$ ) BAD-perylene mixtures of different molar ratios, dissolved in deoxygenated $\mathrm{DCM}\left(\mathrm{C}_{\mathrm{BAD}}=1 \times 10^{-5} \mathrm{M}\right)$.

annihilation of two perylene triplets (Fig. 2a), is taking place. Since the triplet state of $\mathrm{BAD}$ was found to be non-emissive, we used transient absorption spectroscopy measurements to quantify the efficiency of TTET $\left(\phi_{\text {TTET }}\right)$ in the BAD-perylene pair.

Transient absorption data for $\mathrm{BAD}$ and $\mathrm{BAD}$-perylene mixtures are presented in Fig. 4 (see Fig. S1, ESI $\dagger$ for details). The measured triplet state lifetime of BAD in deoxygenated DCM was found to be $246 \mu$ s. Fig. 4 shows that in the presence of perylene the triplet state lifetime of $\mathrm{BAD}$ is strongly decreased due to TTET from BAD to the triplet state of perylene.

$\phi_{\text {TTET }}$ was calculated from eqn (S1, ESI $\dagger$ ). $\phi_{\text {TTET }}$ values of 0.93 and 0.99 , obtained for $1: 1$ and 1:10 mixtures, respectively, indicate that perylene is a very efficient quencher of the BAD triplet state. The BAD triplet state decay could not be measured in the 1:100 mixture due to very fast deactivation of the $T_{1}$ state. This explains the results of Fig. 3b, where a lower than expected increase in the UC intensity was observed with increasing $\mathrm{BAD}$ : perylene ratios. Indeed, even at a 1:10 ratio we observed very efficient TTET and, therefore, saturation of the concentration of perylene triplet states. Thus, any further increase of the perylene concentration has a slightly negative impact; the UC intensity decreases due to reabsorption of emitted photons.

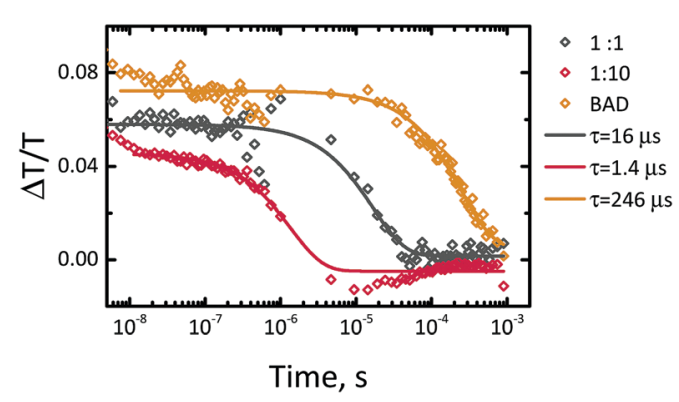

Fig. 4 Decays the BAD ground state bleach (measured at $505 \mathrm{~nm}$ ) in transient absorption experiment for pure $B A D$ and $B A D$-perylene mixtures upon excitation at $355 \mathrm{~nm}$ at a fluence of $0.5 \mathrm{~mJ} \mathrm{~cm}^{-2}$ per pulse. 

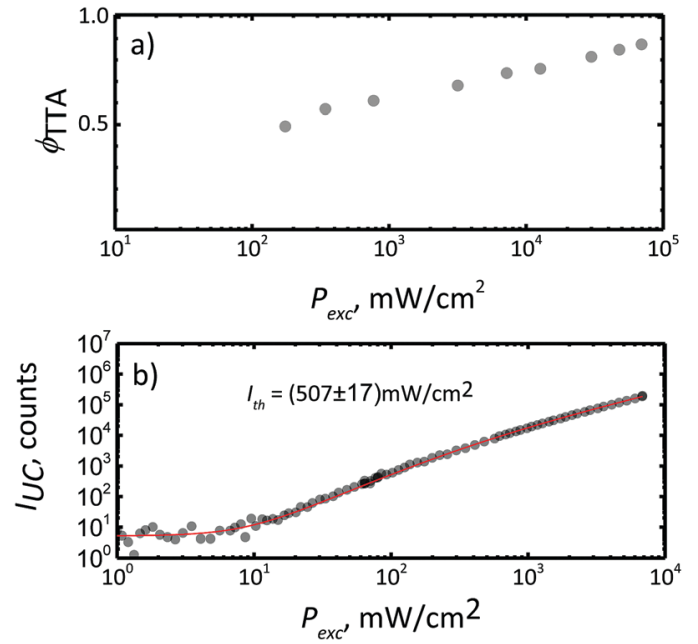

Fig. 5 (a) Dependence of $\phi_{\text {TTA }}$ as function of $P_{\text {exc }}$. (b) Dependence $/ u c$ as function of $P_{\text {exc }} \cdot C_{\mathrm{BAD}}=1 \times 10^{-5} \mathrm{~mol} \mathrm{~L}^{-1}, \mathrm{BAD}$ : perylene ratio $1: 100, \lambda_{\mathrm{exc}}=$ $525 \mathrm{~nm}$.

The efficiency of triplet-triplet annihilation $\left(\phi_{\text {TTA }}\right)$ was estimated via global fitting of the UC decays measured for the fixed $\mathrm{BAD}$ :perylene ratio at variable excitation power density. $\phi_{\mathrm{TTA}}$ was calculated from eqn (S2 and S3) (ESI $\dagger$ ) in line with previous works. $^{13}$

The global fitting of the data presented in Fig. S2 (ESI $\dagger$ ) gives the decay rate of the emitter triplet state $k_{\mathrm{EM}}=0.00067 \mu \mathrm{s}^{-1}$. This corresponds to the lifetime of the perylene triplet state $\tau_{\text {perylene }}^{\mathrm{T}}=1 / k_{\mathrm{EM}}=1.5 \mathrm{~ms}$. The global fitting of the UC decays with eqn (S2, ESI $\dagger$ ) was also used for estimation of the initial fraction of decay that occurs through the TTA $(\beta)$ and calculation of $\phi_{\text {TTA }}$ with eqn (S3, ESI $\dagger$ ). Fig. 5a shows that $\phi_{\text {TTA }}$ grows slowly from 0.6 (at $310 \mathrm{~mW} \mathrm{~cm}^{-2}$ ) to 0.88 (at $70 \mathrm{~W} \mathrm{~cm}^{-2}$ ). This range approximately corresponds to the UC threshold $\left(I_{\mathrm{th}}\right)$ of $507 \mathrm{~mW} \mathrm{~cm}^{-2}$ estimated from the intensity dependence presented in Fig. $5 \mathrm{~b}$ by fitting with eqn (S4, ESI $\dagger) .{ }^{14}$ It is commonly accepted that $n=2$ (in eqn (S5, ESI $\dagger))$ at low excitation limit $\left(P_{\text {exc }}<I_{\text {th }}\right)$ and $n=1$ at high excitation limit $\left(P_{\text {exc }}>I_{\mathrm{th}}\right) \cdot{ }^{14}$ In other words $I_{\mathrm{th}}$ signifies what $P_{\text {exc }}$ is required for relaxation of emitter triplet states mainly via triplet-triplet annihilation.

The obtained value of $I_{\text {th }}$ is rather high for TTA-UC systems. However, we can expect a significant lowering $I_{\text {th }}$ by changing the experimental conditions. $I_{\text {th }}$ can be expressed with eqn (S6, ESI $\dagger$ ). Since $I_{\text {th }}$ is inversely proportional to the number of absorbed photons, an increase in BAD concentration together with the optimal excitation wavelength $(505 \mathrm{~nm})$ provides a straightforward way to reduce the $I_{\mathrm{th}}$ down to tens of $\mathrm{mW} \mathrm{cm}^{-2}$.

As discussed earlier, a change of the solvent polarity dramatically alters the probability of the population of the BAD triplet state. In a non-polar environment, BAD decays preferentially from the singlet state, whereas in polar media it forms a charge-transfer state from which the local triplet state $\mathrm{T}_{1}^{\mathrm{BDP}}$ can be produced. Thus, in low polarity solvents we expect $\mathrm{BAD}$ to function as the UC emitter. To prove this concept we investigated UC in a PdTBTFP-BAD system dissolved in toluene. Fig. 6a shows the absorption spectrum of PdTBTFP with absorption maxima at 440 and $630 \mathrm{~nm}$. The UC
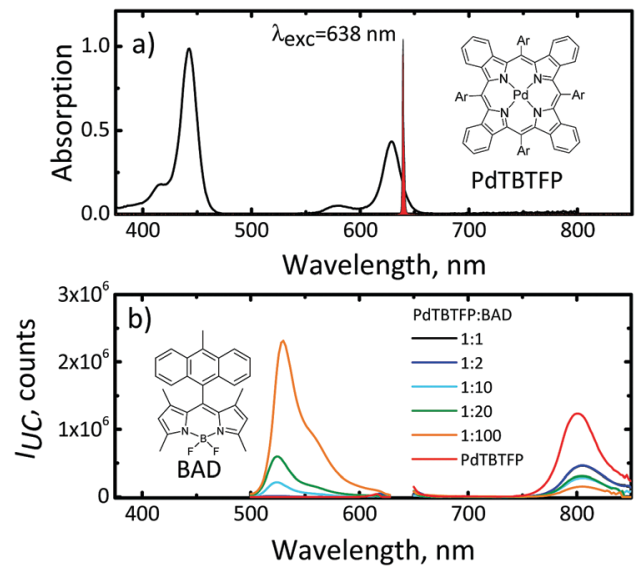

Fig. 6 (a) Normalized absorption spectra of PdTBTFP in toluene and emission profile of a laser diode $(638 \mathrm{~nm})$ used for UC excitation; (b) UC luminescence of BAD under excitation with the $638 \mathrm{~nm}$ laser diode (power density of $9.5 \mathrm{~W} \mathrm{~cm}^{-2}$ ) at different PdTBTFP: BAD ratios dissolved in deoxygenated toluene $\left(C_{\text {PdTBTFP }}=1 \times 10^{-5} \mathrm{M}\right)$.

emission of the PdTBTFP-BAD pair dissolved in deoxygenated toluene was studied upon excitation at $638 \mathrm{~nm}$ for different ratios of PdTBTFP and BAD. PdTBTFP-BAD solutions exhibit UC emission of $\mathrm{BAD}$ with a maximum at $529 \mathrm{~nm}$, whereas the near-infrared emission at $805 \mathrm{~nm}$ corresponds to phosphorescence of PdTBTFP. In order to gain more insight into TTET between PdTBTFP and BAD, we investigated decays of the PdTBTFP phosphorescence. The decay of pure PdTBTFP shows single exponential behavior with a lifetime $\left(\tau_{\text {PdTBтFP }}^{\mathrm{T}}\right)$ of $331 \mu \mathrm{s}$. In contrast, mixtures of PdTBTFP and BAD dissolved in toluene show double exponential behavior with $\tau_{1}<\tau_{\text {PdTBтFP }}^{\mathrm{T}}$ and $\tau_{2}>$

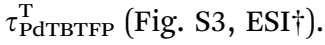

We assume that the short-lived component reflects the lifetime decreasing due to triplet-triplet energy transfer, whereas the long-lived component reflects back-energy transfer from the BAD triplet state to the triplet state of PdTBTFP due to practically equal energy of triplet levels of two molecules. Thus, we estimated initial TTET efficiency (without taking into account the back energy transfer) in agreement with eqn (S7, ESI $\dagger$ ). Fig. 7a displays the dependence of $\phi_{\text {TTET }}$ as a function of BAD concentration. With an increase of BAD concentration we observed a rise of $\phi_{\text {TTET }}$ reaching $70-80 \%$ starting from the ratio $1: 20$.

Using the aforementioned global fitting approach (Fig. S4, $\mathrm{ESI} \dagger) \phi_{\text {TTA }}$ was estimated. The global fitting with eqn (S2, ESI $\dagger$ ) leads to the value of $k_{\mathrm{EM}}=0.00175 \mu \mathrm{s}^{-1}$ or the lifetime of the $\mathrm{BAD}$ triplet state in toluene $\tau_{\mathrm{BAD}}^{\mathrm{T}}=1 / k_{\mathrm{EM}}=571 \mu \mathrm{s}$. This value appears high, but is of the same order of magnitude as the value estimated earlier in DCM via transient absorption measurements. Fig. 7b displays the changes of $\phi_{\mathrm{TTA}}$ as a function of the excitation power density. The value of $\phi_{\text {TTA }}$ grows from 0.14 (at $28 \mathrm{~mW} \mathrm{~cm}^{-2}$ ) to 0.78 (at $74 \mathrm{~W} \mathrm{~cm}^{-2}$ ). This behavior roughly corresponds to $I_{\text {th }}$ estimated from Fig. 7c and eqn (S4, ESI $\dagger$ ) as $31 \mathrm{~mW} \mathrm{~cm}^{-2}$. Finally, we measured UC PLQY according to the technique presented by de Mello et al. ${ }^{15}$ We found that PLQY of the $\mathrm{BAD}$-perylene pair (concentration $10^{-5} \mathrm{M}$ and $10^{-3} \mathrm{M}$, 

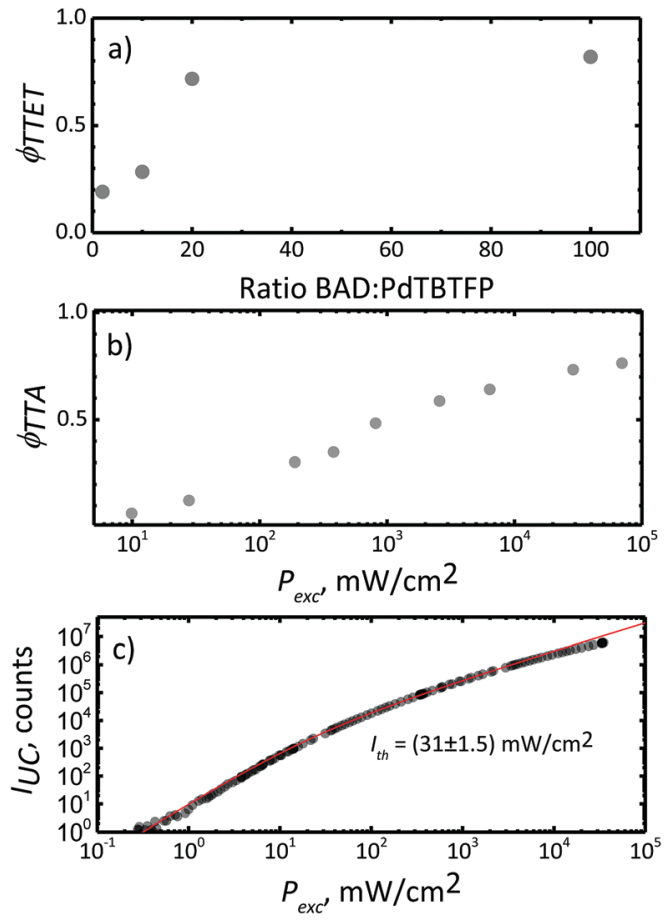

Fig. 7 (a) Dependence of $\phi_{\text {TTET }}$ as function of the PdTBTFP: BAD ratio, $C_{\text {BAD }}=1 \times 10^{-5} \mathrm{~mol} \mathrm{~L}^{-1}$. (b) Dependence of $\phi_{T T A}$ as function of $P_{\text {exc }}$. (c) Dependence $l_{\mathrm{Uc}}$ as function of $P_{\text {exc }} \cdot C_{\mathrm{BAD}}=1 \times 10^{-5} \mathrm{~mol} \mathrm{~L}^{-1}$, PdTBTFP: BAD ratio $1: 100, \lambda_{\text {exc }}=638 \mathrm{~nm}$.

respectively) is $1.2 \pm 0.2 \%$. We explain the large difference to a previously reported PLQY of $\sim 10.1 \%,{ }^{16}$ noting that, firstly, in the previous work PLQY was estimated via the use of a reference fluorescent molecule (not an absolute measurement as in this work). Secondly, PLQY in the work of Wang and Zhao was multiplied by a factor of 2 in order to set the maximum quantum yield to unity. A further difference in our measurements of PLQY is the significant fraction of emitted photons reabsorption. For instance, Fig. S5 (ESI $\dagger$ ) clearly indicates very strong reabsorption for the sample characterized in the integrating sphere.

The PLQY of PdTBTFP-BAD pair (concentration $10^{-5} \mathrm{M}$ and $10^{-3} \mathrm{M}$, respectively) was found to be $1.6 \pm 0.2 \%$. This value is also an underestimation due to reabsorption in the integrating sphere. Another factor limiting UC efficiency in PdTBTFP-BAD is back energy transfer from the BAD triplet to the PdTBTFP triplet, as described earlier. This back transfer reduces $\phi_{\text {TTET }}$ and, thus, the overall PLQY of the system.

To summarize, we report for the first time that a dye can play either the role of sensitizer or emitter in TTA-UC depending on polarity of the solvent. In non-polar toluene the BAD dyad acts as the emitter; it emits green light with $\lambda_{\max }=530 \mathrm{~nm}$ after excitation of PdTBTFP at $638 \mathrm{~nm}$. In polar DCM the BAD dyad acts as the sensitizer. Excitation of the BAD dyad at $525 \mathrm{~nm}$ results blue emission of perylene with $\lambda_{\max }=445 \mathrm{~nm}$. This unique behavior is due to changing of charge-transfer and local excited state relative energy ordering in the dyad by polarity of the media. The aforementioned effect was also observed employing other polar and non-polar solvents. The scope of dyads capable of such behaviour is broad as other aromatic electron donors can be combined with BODIPY, thereby allowing absorption and emission profiles to be shifted. Studies of such systems are on the way and will be reported in due course.

M. A. F. acknowledges the European Commission (CONSORT, Grant No. 655142). The authors would like to acknowledge the financial support provided by the Helmholtz Association: (i) a Recruitment Initiative Fellowship for B. S. R.; (ii) the funding of chemical synthesis equipment from the Helmholtz Materials Energy Foundry (HEMF); and (iii) the Science and Technology of Nanosystems research program. M. O. S. acknowledges support by Science Foundation Ireland (SFI IvP 13/IA/1894); N. K. acknowledges a DAAD scholarship.

\section{Conflicts of interest}

There are no conflicts to declare.

\section{References}

1 J. Zhou, Q. Liu, W. Feng, Y. Sun and F. Li, Chem. Rev., 2015, 115, 395-465. 2 R. Medishetty, J. K. Zareba, D. Mayer, M. Samoc and R. A. Fischer, Chem. Soc. Rev., 2017, 46, 4976-5004.

3 L. Frazer, J. K. Gallaher and T. W. Schmidt, ACS Energy Lett., 2017, 2, 1346-1354.

4 V. Gray, D. Dzebo, M. Abrahamsson, B. Albinsson and K. Moth-Poulsen, Phys. Chem. Chem. Phys., 2014, 16, 10345-10352.

5 S. H. C. Askes, W. Pomp, S. L. Hopkins, A. Kros, S. Wu, T. Schmidt and S. Bonnet, Small, 2016, 12, 5579-5590.

6 T. F. Schulze and T. W. Schmidt, Energy Environ. Sci., 2015, 8, 103-125.

7 D. V. Kozlov and F. N. Castellano, Chem. Commun., 2004, 2860-2861.

8 M. A. Filatov, F. Etzold, D. Gehrig, F. Laquai, D. Busko, K. Landfester and S. Baluschev, Dalton Trans., 2015, 44, 19207-19217.

9 D. Dzebo, K. Börjesson, V. Gray, K. Moth-Poulsen and B. Albinsson, J. Phys. Chem. C, 2016, 120, 23397-23406.

$10 \mathrm{~J}$. R. Lakowicz, Principles of fluorescence spectroscopy, Springer Science + Business Media, LLC, Boston, MA, 2006.

11 Z. Yang, Z. Mao, Z. Xie, Y. Zhang, S. Liu, J. Zhao, J. Xu, Z. Chi and M. P. Aldred, Chem. Soc. Rev., 2017, 46, 915-1016.

12 M. A. Filatov, S. Karuthedath, P. M. Polestshuk, H. Savoie, K. J. Flanagan, C. Sy, E. Sitte, M. Telitchko, F. Laquai, R. W. Boyle and M. O. Senge, J. Am. Chem. Soc., 2017, 139, 6282-6285.

13 Y. Y. Cheng, B. Fückel, T. Khoury, R. G. C. R. Clady, M. J. Y. Tayebjee, N. J. Ekins-Daukes, M. J. Crossley and T. W. Schmidt, J. Phys. Chem. Lett., 2010, 1, 1795-1799.

14 A. Monguzzi, R. Tubino, S. Hoseinkhani, M. Campione and F. Meinardi, Phys. Chem. Chem. Phys., 2012, 14, 4322-4332.

15 J. C. de Mello, H. F. Wittmann and R. H. Friend, Adv. Mater., 1997, 9, 230-232.

16 Z. Wang and J. Zhao, Org. Lett., 2017, 19, 4492-4495. 